University of Wollongong

Research Online

Australian Institute for Innovative Materials -

Papers

Australian Institute for Innovative Materials

$1-1-2011$

Oxidative acetylenic coupling reactions as a surface chemistry tool

Simone Ciampi

University of New South Wales, sciampi@uow.edu.au

Michael James

University of New South Wales

Nadim A. Darwish

University of New South Wales, nadimdarwish@gmail.com

Erwann Luais

University of New South Wales

Bin Guan

University of New South Wales

See next page for additional authors

Follow this and additional works at: https://ro.uow.edu.au/aiimpapers

Part of the Engineering Commons, and the Physical Sciences and Mathematics Commons

Research Online is the open access institutional repository for the University of Wollongong. For further information contact the UOW Library: research-pubs@uow.edu.au 


\title{
Oxidative acetylenic coupling reactions as a surface chemistry tool
}

\begin{abstract}
A novel method to prepare redox monolayers on silicon electrodes has been developed that employs Culcatalyzed oxidative acetylenic coupling reactions for molecular electronic type applications. As the first case study, ethynylferrocene was covalently immobilized onto an acetylene-terminated monolayer on a $\mathrm{Si}(100)$ surface to give a 1,3-diyne (CC-CC-) linked redox assembly. The derivatization process requires no protection/de-protection steps, nor activation procedures. The effect of the conjugated diyne linkage on the rate of electron transfer between tethered ferrocenyl units and the silicon electrode is benchmarked against well-established "click" products (i.e. 1,2,3-triazole linkage). The surfaces, after each step, are characterized thoroughly using X-ray reflectivity (XRR), X-ray photoelectron spectroscopy (XPS), electrochemical impedance spectroscopy (EIS) and cyclic voltammetry (CV). The coupling chemistry provides a useful strategy for functionalizing silicon surfaces and contributes to an expanding repertoire of wet chemistry routes for the functionalization of solid substrates. This journal is the Owner Societies.
\end{abstract}

\section{Keywords}

oxidative, acetylenic, coupling, reactions, chemistry, surface, tool

\section{Disciplines}

Engineering | Physical Sciences and Mathematics

\section{Publication Details}

Ciampi, S., James, M., Darwish, N., Luais, E., Guan, B., Harper, J. B. \& Gooding, J. Justin. (2011). Oxidative acetylenic coupling reactions as a surface chemistry tool. Physical Chemistry Chemical Physics, 13 (34), 15624-15632.

\section{Authors}

Simone Ciampi, Michael James, Nadim A. Darwish, Erwann Luais, Bin Guan, Jason Brian Harper, and J Justin Gooding 


\title{
Oxidative acetylenic coupling reactions as a surface chemistry tool $\dagger$
}

\author{
Simone Ciampi, ${ }^{a}$ Michael James, ${ }^{a b}$ Nadim Darwish, ${ }^{a}$ Erwann Luais, ${ }^{a}$ Bin Guan, ${ }^{a}$ \\ Jason B. Harper ${ }^{a}$ and J. Justin Gooding*a
}

Received 6th May 2011, Accepted 8th July 2011

DOI: $10.1039 / \mathrm{c} 1 \mathrm{cp} 21450 \mathrm{k}$

A novel method to prepare redox monolayers on silicon electrodes has been developed that employs $\mathrm{Cu}^{\mathrm{I}}$-catalyzed oxidative acetylenic coupling reactions for molecular electronic type applications. As the first case study, ethynylferrocene was covalently immobilized onto an acetylene-terminated monolayer on a $\mathrm{Si}(100)$ surface to give a 1,3 -diyne $(\mathrm{C} \equiv \mathrm{C}-\mathrm{C} \equiv \mathrm{C}-)$ linked redox assembly. The derivatization process requires no protection/de-protection steps, nor activation procedures. The effect of the conjugated diyne linkage on the rate of electron transfer between tethered ferrocenyl units and the silicon electrode is benchmarked against well-established "click" products (i.e. 1,2,3-triazole linkage). The surfaces, after each step, are characterized thoroughly using X-ray reflectivity (XRR), X-ray photoelectron spectroscopy (XPS), electrochemical impedance spectroscopy (EIS) and cyclic voltammetry (CV). The coupling chemistry provides a useful strategy for functionalizing silicon surfaces and contributes to an expanding repertoire of wet chemistry routes for the functionalization of solid substrates.

\section{Introduction}

Compared with other functional groups, the chemical reactivity diversity and richness of the alkyne functional group has arguably no equal. ${ }^{1}$ The synthetic versatility of either 1-alkynes, ${ }^{2}$ functionalized acetylenes and acetylenes derivatives (e.g. 1-cyano ${ }^{3}$ and 1-halogenoacetylenes, ${ }^{4}$ alkyniliodonium salts, ${ }^{5}$ and phosphaalkynes ${ }^{6}$ ), or metal- $\pi$-bonded alkyne complexes, ${ }^{7}$ account for the central role played by acetylenes as "building blocks" in fields such as supramolecular chemistry, ${ }^{8}$ material science, ${ }^{9}$ energy research, ${ }^{10}$ molecular biology, ${ }^{11}$ cell biology, ${ }^{12}$ medicinal chemistry, ${ }^{13}$ and nanotechnology. ${ }^{14}$

Quite surprisingly, despite the wide and diverse use of acetylene "building blocks" in solution-phase chemistry, ${ }^{15}$ there are few published studies that have reported on the unique properties of the carbon-carbon triple bond in the context of derivatizing solid substrates. Notably, only $\mathrm{Cu}^{\mathrm{I}}$-catalyzed alkyne-azide cycloaddition $(\mathrm{CuAAC})$ reactions have been extensively explored as a tool to introduce precise functionalities onto a solid surface. ${ }^{2 c, d, 16}$ Only few other examples of acetylenic coupling reactions onto solid substrates are found in the literature. ${ }^{17}$

${ }^{a}$ School of Chemistry, The University of New South Wales, Sydney, NSW 2052,Australia.E-mail: justin.gooding@unsw.edu.au; Fax: 61-2-9385 5384; Tel: 61-2-9385 6141

${ }^{b}$ Bragg Institute, Australian Nuclear Science and Technology Organisation (ANSTO), Locked Bag 2001,

Kirrawee DC NSW 2232, Australia

$\dagger$ Electronic supplementary information (ESI) available: experimental procedures, additional electrochemical and spectroscopic data. See DOI: $10.1039 / \mathrm{clcp} 21450 \mathrm{k}$
As comprehensively reviewed elsewhere, ${ }^{18}$ formation of molecular layers on crystalline silicon surfaces without an intervening oxide layer is an extremely appealing approach toward robust layers on a surface. ${ }^{19}$ Both 1-alkenes and 1-alkynes can be used for monolayer assembly on hydrogenated silicon surfaces $(\mathrm{Si}-\mathrm{H}) .{ }^{18 d, 19 a, 20}$ Recent research suggests not only faster monolayer formation with 1-alkynes but also a more stable and densely packed organic monolayer. ${ }^{21}$ Insertion of 1-alkynes into a silicon-hydrogen bond results in a 1-en-1-yl silyl $(\mathrm{Si}-\mathrm{C}=\mathrm{C})$ linkage, ${ }^{22}$ which may play a significant role in terms of oxidation-inhibiting properties of the alkenyl-grafted monolayer. ${ }^{19 b}$

Silicon-carbon linked monolayers on silicon substrates are expected to complement and/or extend the applications of the currently most relevant technological material ${ }^{23}$ toward the development of atomic scale assemblies ${ }^{24}$ diverse molecular devices, ${ }^{25}$ and well-defined sensing interfaces. ${ }^{16 c, 26}$

The 1,3-diyne linker $(-\mathrm{C} \equiv \mathrm{C}-\mathrm{C} \equiv \mathrm{C}-)$ has recently attracted a great deal of interest as a key element in the preparation of molecular wires or more complex molecular architectures. $^{8,27}$ A broad range of strategies exists for its preparation, with the Glaser and Cadiot-Chodkiewicz coupling reactions being the two major methods. The Glaser coupling, and its variants (e.g. Hay ${ }^{28}$ and Eglinton ${ }^{29}$ reactions), is arguably the oldest and most studied example of oxidative acetylenic coupling. ${ }^{2 h}$ In this paper we report results on the use of oxidative acetylenic coupling reactions to derivatize $\omega$-functionalized, and $\mathrm{Si}-\mathrm{C}=\mathrm{C}$ linked, organic monolayers prepared on $\mathrm{Si}(100)$ substrates. Oxidative coupling represents an efficient alternative to known two-step 

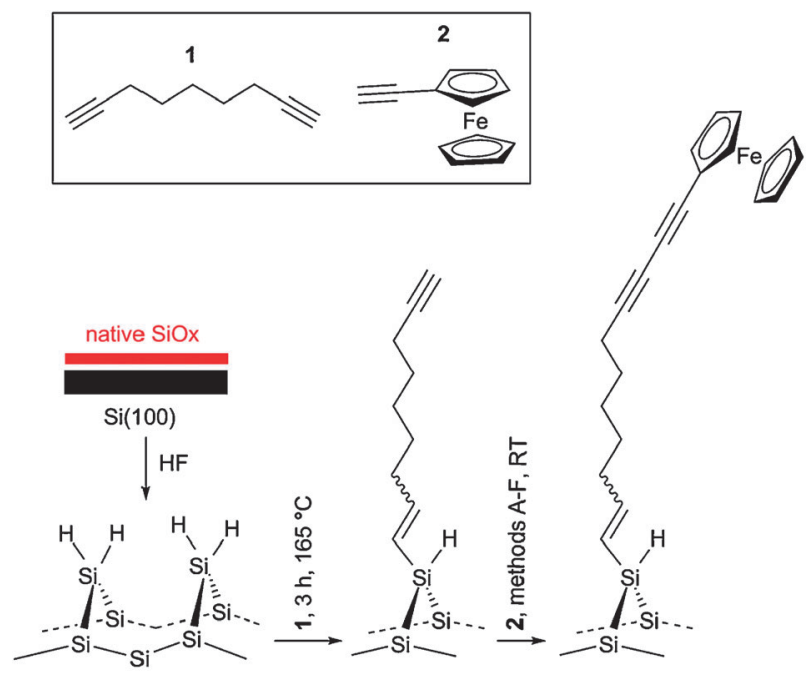

SAM-1

Scheme 1 Molecular assembly on H-Si(100). Direct coupling of acetylene-terminated alkenyl monolayers with 1-alkynes.

methods, such as those involving alkyne halogenation followed by $\mathrm{C}-\mathrm{C}$ cross-coupling. ${ }^{1 b, 17 d}$ Acetylene-terminated $\mathrm{Si}(100)$ monolayers prepared from 1,8-nonadiyne (1; Scheme 1) have previously allowed for the immobilization of solution phase azides via $\mathrm{CuAAC}$ reactions with satisfactory conversion of the surface bound alkyne. ${ }^{30}$ This chemistry effectively protects the silicon from oxidation, ${ }^{30 b}$ and has allowed for unprecedented applications of $\mathrm{Si}(100)$ in electrochemistry, ${ }^{30 c, e, g}$ bioelectronics, ${ }^{30 d}$ and (bio)sensing, ${ }^{31}$ Here we seek to expand the scope of this platform by investigating the use of metalcatalyzed acetylenic cross coupling reactions to immobilize a representative terminal acetylene compound, ethynylferrocene (2; Scheme 1), onto alkyne-terminated monolayers prepared from diyne 1. This is important because the putative product of the alkyne heterocoupling reaction is expected to be a redox-active monolayer with significant conjugation, where the maintenance of distal triple bonds is of interest for both improved rigidity, and improved electronic coupling. ${ }^{32}$

\section{Results and discussion}

The covalent derivatization of the $\mathrm{Si}-\mathrm{H}$ substrate is illustrated in Scheme 1. The X-ray photoelectron spectroscopy (XPS) analyses of samples of SAM-1 support the assertion that heat ${ }^{33}$ greatly facilitates the reaction between a terminal acetylene group and the hydrogen-terminated $\mathrm{Si}(100)$ surface. ${ }^{30 c}$ The immersion of freshly etched hydrogen-terminated $\mathrm{Si}(100)$ surfaces in 1,8-nonadiyne 1 at $165{ }^{\circ} \mathrm{C}$ for $3 \mathrm{~h}$ afforded the acetylene-functionalized surface (SAM-1). The water contact angle for the acetylene-terminated surface was $82 \pm 6^{\circ}$ which is consistent with previously observed values. ${ }^{30 h}$ XPS spectra acquired on SAM-1 are shown in Fig. S1 (Electronic supplementary information, ESI $\dagger$ ). Fig. S1a shows a representative survey spectrum that indicates the presence of $\mathrm{Si}, \mathrm{C}$, and $\mathrm{O}$ only. As suggested by Baio et al. ${ }^{34}$ and James et al., ${ }^{30 \mathrm{~h}}$ the oxygen detected is most likely arising from the presence of oxygenated co-adsorbates, such as tightly-bound water. Highresolution narrow scans were collected for the $\mathrm{C} 1$ s and $\mathrm{Si} 2 \mathrm{p}$ regions to gain information on bonding configurations and determine the extent of any oxidation of the silicon substrate. The narrow scan of the $\mathrm{C} 1 \mathrm{~s}$ region (Fig. S1b) shows a broad signal $(1.6 \mathrm{eV}$ fwhm) with a mean binding energy of $285.0 \mathrm{eV}$ for the fitted function and agrees well with previous data. ${ }^{30 b, d}$ The high-resolution Si 2 p scan reveals important information about the monolayer quality and its ability to prevent appreciable oxidation of the underlying Si substrate. ${ }^{35}$ Most importantly, in Si 2p narrow scans (Fig. S1c) obtained for SAM-1 no silicon oxide was detected ${ }^{36}$ in the $102-104 \mathrm{eV}$ region on the high-binding-energy side of the $\mathrm{Si} 2 \mathrm{p}_{1 / 2}-\mathrm{Si} 2 \mathrm{p}_{3 / 2}$ spin-orbitsplit doublet (99.9 and $99.3 \mathrm{eV}$, respectively). ${ }^{37}$

Next the surfaces were explored with X-ray reflectivity (XRR) to obtain the thickness $(d)$ of such films to be measured with atomic resolution, as made possible by the high contrast in scattering length density (SLD) ${ }^{38}$ of the organic molecules in the SAM (SLD $\sim 10 \times 10^{-6} \AA^{-2}$ ) compared to air $(\mathrm{SLD}=0)$ and the silicon substrate $\left(\mathrm{SLD}=20.1 \times 10^{-6} \AA^{-2}\right) .{ }^{30 h}$ The film thickness, in concert with the refined SLD, can also be used to establish the surface area/molecule in the monolayer. The average surface roughness $(\sigma)$ of these monolayers can also be precisely determined using this technique. Fig. 1 shows the measured specular XRR for SAM-1 and SAM-2 samples (Pd- and Cu-catalyzed reactions, methods $\mathrm{A}$ and $\mathrm{F}$ respectively). The solid lines are theoretical fits to the experimental data. ${ }^{39}$ Unlike the Fresnel-like decay of the H-Si(100) surface (Fig. S2, ESI $\dagger$ ), samples with organic films show clear interference thickness oscillations (Fig. 1a-c). The best fit for the $\mathrm{H}-\mathrm{Si}(100)$ sample has a surface roughness $\left(\sigma_{\mathrm{Si}}\right)$ of $1.7(1) \AA{ }^{40}$ which is typical for high-quality silicon substrates. ${ }^{30 h, 41}$

The reflectivity spectra from SAM-1 samples were simulated using a one-layer model (Fig. 1a) and Table 1 summarizes the results. Our XRR-determined SAM-1 film thickness of 9.6(1) $\AA$ is close to the $10-12 \AA$ that was previously reported. ${ }^{30 b, c}$ The electron density $\left(\rho_{\mathrm{el}}\right)$ of the organic layer was also derived from the X-ray reflectivity data and was found to be $c a .0 .37 \mathrm{e}^{-} / \AA^{3}$, consistent with previous reports of alkane-based SAMs. This electron density suggests the formation of a densely packed organic layer with very few defects. ${ }^{19 a, 41,42}$ On the basis of both the refined values of monolayer thickness and electron density, an estimate of the molecular coverage of the silicon surface of 19(3) $\AA^{2}$ per grafted molecule of diyne $\mathbf{1}$ is proposed. Both the refined silicon-organic $\left(\sigma_{\mathrm{Si}}\right)$ and monolayer-air $\left(\sigma_{\mathrm{ML}}\right)$ interfacial roughnesses were found to be $c a$. 2-3 $\AA$, which reflects a good-quality monolayer film. . $^{30,41,43}$

As documented in the literature, the predominant formation of the symmetrical 1,3-diyne product (i.e. homocoupled product) in the oxidative dimerization of terminal acetylenes has hampered the use of the Glaser reaction, as well as its variants (e.g. Hay reaction), in wet chemistry heterocoupling reactions. ${ }^{2 h}$ Unsymmetrical bis-acetylenes are routinely prepared by using the Cadiot-Chodkiewicz reaction. ${ }^{44}$ In that method, a terminal acetylene is reacted with a bromoalkyne. In contrast to solution phase chemistry, the use of tethered terminal acetylenes, i.e. SAM-1, gave us rapid access to a purified unsymmetrical 1,3-diyne on a surface (SAM-2), thus accelerating the screening process of the reaction conditions for this first example of oxidative acetylenic coupling at a surface. Further, no synthetic effort was required for the preparation 
a)

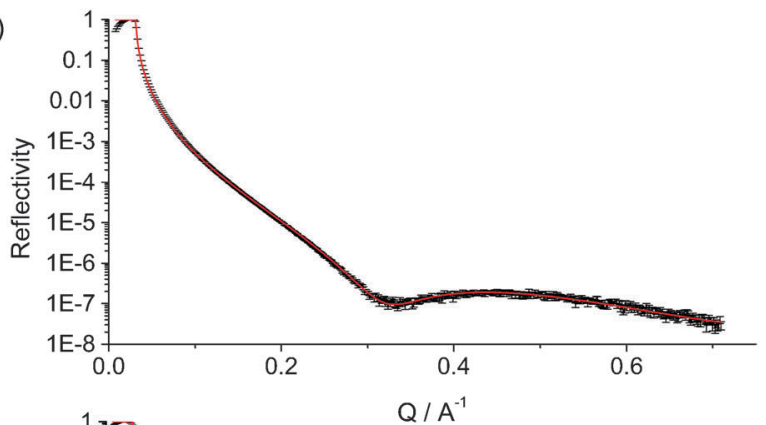

b)

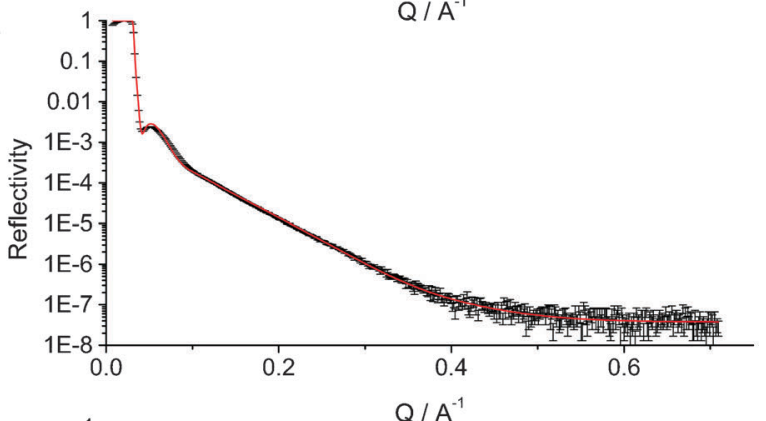

c)

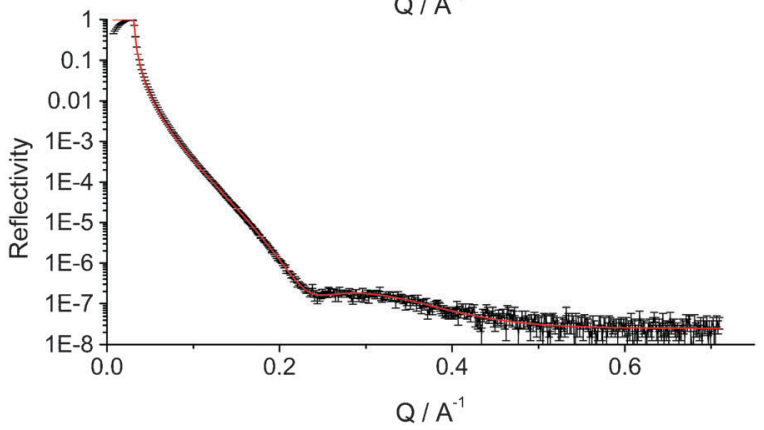

Fig. 1 X-ray reflectivity spectra acquired on organic modified $\mathrm{Si}(100)$ surfaces. (a) Acetylene-terminated monolayers (SAM-1). (b) Ethynylferrocene-derived sample prepared under palladium-catalyzed oxidative coupling conditions (SAM-2, method A). (c) Copper-catalyzed immobilization of ethynylferrocene (SAM-2, method F). The refinement of a structural model is shown as the solid line.

Table 1 Refined structural parameters from XRR data ${ }^{a}$

\begin{tabular}{lllll}
\hline & & \multicolumn{3}{c}{ SAM-2 $^{b}$} \\
& H-Si(111) & SAM-1 & layer 1/layer 2 & SAM-2 $^{c}$ \\
\hline$\sigma_{\mathrm{Si}}(\AA)$ & $1.7(1)$ & $1.7(3)$ & $3.28(8)$ & $2.9(7)$ \\
$\sigma_{\mathrm{ML}}(\AA)$ & - & $3.2(3)$ & $4.8(3) / 31.5(9)$ & $7.8(7)$ \\
$d(\AA)$ & - & $9.6(1)$ & $15.0(3) / 75.8(14)$ & $12.0(9)$ \\
$\mathrm{SLD}\left(\times 10^{-6} \AA^{-2}\right)$ & - & $10.4(1)$ & $9.2(2) / 13.6(2)$ & $14.9(8)$
\end{tabular}

${ }^{a}$ Estimated standard deviations (esd's) are given in parentheses. ${ }^{b} \mathrm{Pd}-$ catalyzed reaction (method A). ${ }^{c} \mathrm{Cu}$-catalyzed reaction (method F).

of the bromo derivative of the alkyne 2 , and the presence of a redox centre at the distal end of the putative heterocoupled product (SAM-2) allowed for a convenient analytical tool in the evaluation of isolated reaction yields. Electrochemical detection by cyclic voltammetry $(\mathrm{CV})$ of surface-bound ferrocenyl units was used here to monitor the reaction progress and assess rapidly the extent of conversion of surface alkyne moieties.

The robustness of oxidative acetylenic coupling reactions and their compatibility with most functional groups, solvents, organic bases - regardless of the source of the catalyst - is evidenced by the number of experimental conditions that have been successfully employed in literature. ${ }^{1 a, 2 h}$ In our preliminary screening study we opted for variations of the most commonly used, and most efficient, published experimental protocols. Table 2 gives an overview of selected screening results for the preparation of the redox-active 1,3-diyne product SAM-2.

Unless specified otherwise, during the course of the optimization study detailed in the following sections, reactions yields were evaluated by electrochemical methods only (cyclic voltammetry, CV). Nevertheless, a detailed electrochemical and spectroscopic (electrochemical impedance spectroscopy, XPS and XRR) characterization study is presented for the putative surface product obtained under optimized reaction conditions.

\subsection{Oxidative acetylenic heterocoupling at modified Si(100) surfaces: optimization of the reaction method}

A number of different copper(I), palladium(II) and palladium(0) sources can be utilized in the oxidative dimerization of terminal acetylenes. Compared with the copper-mediated (e.g. $\mathrm{CuCl}, \mathrm{CuI}$ ) coupling reactions of terminal alkynes, the palladium-catalyzed transformations are arguably more mild, efficient, and chemoselective. ${ }^{45}$ Moreover, the palladiumcatalyzed reactions of aliphatic alkynes, as well as aromatic alkynes, are known to afford good to excellent yields, whereas low to moderate yields are often obtained when homocoupling of aliphatic alkynes was examined under the Hay reaction conditions. ${ }^{46}$ For the palladium(II)-catalyzed homocoupling reactions of terminal alkynes, several mild and efficient catalytic conditions exist. Ligand-free palladium-catalyzed homocoupling reactions under aerobic conditions have been developed by Xie and co-workers and been shown to result in moderate to excellent yields using air as the reoxidant. ${ }^{45}$ This protocol not only tolerates a range of functional groups but also does not require any additives such as phosphine ligands. In order to optimize the reaction conditions for the attachment of alkyne 2 onto acetylenyl Si(100) monolayers (SAM-1) an analogous Pd-catalyzed protocol was initially evaluated (method A). Despite rigorous rinsing procedures being routinely applied to the derivatized surface prior to analysis of the sample, XPS data suggested the presence of significant levels of iodine-based contaminants ( $c a$. 618 and $630 \mathrm{eV}$ ), presumably due to residual catalyst adsorbed onto the modified silicon surface (wide scan, Fig. S3a, ESI $\dagger$ ). The high resolution XPS scan of the Fe $2 p$ region (Fig. S3b) showed the two major spin-orbit-split components $\mathrm{Fe} 2 \mathrm{p}_{3 / 2}$ and $\mathrm{Fe} 2 \mathrm{p}_{1 / 2}$ at 708.4 and $721.0 \mathrm{eV}$, respectively, thus suggesting a major $\mathrm{Fe}^{\mathrm{II}}$ population with only minor ${ }^{47}$ high-binding energy satellite signals due to $\mathrm{Fe}^{\mathrm{III}}$ species. The presence of $\mathrm{Fe}^{\mathrm{III}}$-related species indicates there was a sizable oxidation of the ferrocene units to ferricenium species under the reaction conditions used. ${ }^{48}$ As shown Fig. S3c, Si 2p high-resolution scans of SAM-2 (method A) suggested partial oxidation of the underlying silicon substrate (ca. 0.1 to $0.2 \mathrm{SiO}_{x}$ fractional monolayers ${ }^{35 a}$ ) upon the oxidative coupling of alkyne 2. Further, as shown in Fig. S4 (ESI $\dagger$ ), the redox behaviour of the putative surface product SAM-2 (method A, Table S1 in ESI $\dagger$ ) was far from ideal (i.e. large dispersion values, $\Delta E_{\mathrm{fwhm}}$, and slow kinetics of electron transfer, $k_{\text {et }}^{\circ}$ ). Surprisingly, measured figures relative to the surface 
Table 2 Selected screening results for palladium- and copper-mediated oxidative coupling of ethynylferrocene 2 at acetylene-terminated Si(100) electrodes (SAM-1)

\begin{tabular}{|c|c|c|c|c|}
\hline Entry/method & Reaction conditions (equiv of reagents) & Solvent/oxidant & Time & Extent of conversion $(\%)^{a, b}(\mathrm{SAM}-2)$ \\
\hline $1 /$ method A & $\mathrm{Pd}(\mathrm{OAc})_{2}(0.1), \mathrm{CuI}(0.1), \mathrm{DABCO}(1.1), 2(20 \mathrm{mM})$ & $\mathrm{MeCN} /$ air & $10 \mathrm{~min}$ & multilayers $230-590$ \\
\hline $2 /$ method A & $\mathrm{Pd}(\mathrm{OAc})_{2}(0.1), \mathrm{CuI}(0.1), \mathrm{DABCO}(1.1), 2(20 \mathrm{mM})$ & $\mathrm{MeCN} /$ air & $30 \mathrm{~min}$ & multilayers $>1350$ \\
\hline $3 /$ method B & $\mathrm{CuCl}(0.3), 2(20 \mathrm{mM})$ & $\mathrm{Py} / \mathrm{O}_{2}$ & $3 \mathrm{~h}$ & not formed \\
\hline 4/method B & $\mathrm{CuCl}(0.3), 2(20 \mathrm{mM})$ & $\mathrm{Py} / \mathrm{air}$ & $3 \mathrm{~h}$ & not formed \\
\hline 5/method C & $\mathrm{CuCl}$ (1.3), TMEDA (2.6), 2 (20 mM) & $\mathrm{DCM} / \mathrm{O}_{2}$ & $3 \mathrm{~h}$ & not formed \\
\hline 6/method D & $\mathrm{CuCl}$ (3), TMEDA (13), 2 (0.7 mM) & $\mathrm{MeCN} / \mathrm{O}_{2}$ & $45 \mathrm{~min}$ & 0.3 \\
\hline 7/method D & $\mathrm{CuCl}$ (3), TMEDA (13), 2 (0.7 mM) & $\mathrm{MeCN} /$ air & $45 \mathrm{~min}$ & $0.3 \pm 0.1$ \\
\hline 8/method E & $\mathrm{CuCl}(3)$, TMEDA (13), DABCO (13), 2 (0.7 mM) & $\mathrm{MeCN} / \mathrm{air}$ & $45 \min$ & $0.9 \pm 0.1$ \\
\hline $9 /$ method E & $\mathrm{CuCl}(3)$, TMEDA (13), DBU (15), 2 (0.7 mM) & $\mathrm{MeCN} /$ air & $45 \mathrm{~min}$ & 0.8 \\
\hline $10 /$ method F & $\mathrm{CuCl}(3), \operatorname{DABCO}(13), 2(0.7 \mathrm{mM})$ & $\mathrm{MeCN} /$ air & $45 \mathrm{~min}$ & $2.0 \pm 0.3$ \\
\hline $11 /$ method F & $\mathrm{CuCl}(3), \mathrm{DABCO}(13), 2$ (20 mM) & $\mathrm{MeCN} / \mathrm{air}$ & $45 \mathrm{~min}$ & $4.7 \pm 1.9$ \\
\hline $12 / \operatorname{method} \mathrm{F}$ & $\mathrm{CuCl}(0.1), \mathrm{DABCO}(1.1), 2(20 \mathrm{mM})$ & $\mathrm{MeCN} /$ air & $45 \mathrm{~min}$ & $3.9 \pm 1.9$ \\
\hline $13 /$ method F & $\mathrm{CuCl}(0.1), \mathrm{DABCO}(1.1), 2(20 \mathrm{mM})$ & $\mathrm{MeCN} / \mathrm{air}$ & $24 \mathrm{~h}$ & $4.8 \pm 2.1$ \\
\hline $14 /$ control & $\mathrm{CuCl}(0.1)$, DABCO (1.1), vinylferrocene $(20 \mathrm{mM})$ & $\mathrm{MeCN} / \mathrm{air}$ & $45 \mathrm{~min}$ & not formed \\
\hline $15 /$ control & $2(20 \mathrm{mM})$ & $\mathrm{MeCN} /$ air & $45 \mathrm{~min}$ & not formed \\
\hline $16 /$ control & DABCO (1.1), $2(20 \mathrm{mM})$ & $\mathrm{MeCN} /$ air & $45 \mathrm{~min}$ & not formed \\
\hline
\end{tabular}

${ }^{a}$ Electrochemically measured (CVs) reaction yields. ${ }^{b}$ Estimated standard deviations are reported for entries obtained from the analysis of three or more independently prepared and analyzed surface samples.

density of redox centers were most discouraging. From an analysis of the area under each of the CV's cathodic and anodic peaks (Fig. S4a, ESI $\dagger$ ), the measured coverage of redox-active ferrocenyl units, $\Gamma$, for SAM-2 was found between $1.1 \times 10^{-8}$ and $1.9 \times 10^{-9} \mathrm{~mol} \mathrm{~cm}^{-2}$. The obtained $\Gamma$ values are significantly higher than those reported for densely packed ferrocene films obtained by direct attachment of either ethynylferrocene $\mathbf{2}$ or vinylferrocene onto hydrogenterminated $\mathrm{Si}(100)\left(\mathrm{ca} .1 \times 10^{-10} \mathrm{~mol} \mathrm{~cm}^{-2}\right) .{ }^{49}$ Considering the surface density of silicon top sites on $\mathrm{Si}(100), 6.78 \times 10^{14} \mathrm{~cm}^{-2}, 50$ the electrochemically measured density of redox sites in the film yields a silicon atom/ferrocene molecule ratio of $c a .0 .1-0.6{ }^{51}$ From the perspective that surface acetylene groups have a footprint of 19(3) $\AA^{2}$ (refinement of XRR structural model for surface SAM-1, see above) electrochemical data thus suggest conversions between 230 and $1350 \%$. We can thus infer that under the conditions of method $\mathrm{A}$, the surface reaction is poorly controlled, yielding a multilayer system rather than the putative monolayer product (SAM-2). Furthermore, the poor quality of the electrode surface (SAM-2) prepared via the Pd-catalyzed attachment of alkyne $\mathbf{2}$ onto the acetyleneterminated monolayer was evidenced in the limited stability of its voltammetric response, with a loss of electroactive ferrocenyl units as great as $25 \%$ over the first $50 \mathrm{CV}$ cycles.

To further detail the formation of a structurally poorly defined organic multilayer system under these initial and not optimized $\mathrm{Pd}(\mathrm{OAc})_{2}$-catalyzed reaction conditions (method A), $\mathrm{X}$-ray reflectivity measurements were performed on SAM-2.

Experimental reflectivity data were fitted to a bilayer structural model (Fig. 1b), with the refined structural parameters summarized in Table 1. The appearance of the interference minimum at a lower value of $Q$ for the SAM-2 sample indicates that it has a thicker film than the SAM-1 monolayer. The thickness of the inner layer was 15.0(3) $\AA$, which is in modest agreement with the refined values obtained from surface SAM-1 (Fig. 1a). The thickness of the outer layer was found to be $75.8(14) \AA$, with a refined total thickness of the organic layer of $c a .91 \AA$. XRR data are consistent with a significant conversion of the acetylenyl surface (SAM-1) to a structurally ill-defined (ca. 32 A surface roughness) multilayer system. These results are consistent with CV data and indicate that the derivatization of the distal acetylene of SAM-1 using method A (Table 1, entries 1 and 2) is not a viable approach toward a chemically well-defined and electrically well-behaved 1,3-diyne derivative. The molecular details of this multilayer system, as well as the mechanism of its formation, are unclear at present and will need further investigation.

As many ethynyl compounds, including ferrocenyl and azaferrocenyl derivatives, ${ }^{52}$ are oxidatively dimerized rapidly at room temperature in almost quantitative yield using amine complexes of copper(I) chloride and either air or oxygen as the oxidant (i.e. Hay modification of the Glaser reaction), ${ }^{28,53}$ we then turned our attention to applying this method (method B). Monodentate tertiary amine solvents such as pyridine usually function satisfactorily, ${ }^{b, 2 h}$ but as summarized in Table 2 (entries 3 and 4) the reaction between the acetylenic substrate (SAM-1) and compound 2 did not proceed at all. ${ }^{54}$ Analogously, our third attempt (method C) to synthesize SAM-2 involving well-established reaction conditions, i.e. adding two equivalent (with regards to $\mathrm{CuCl}$ ) of $N, N, N^{\prime}, N^{\prime}$-tetramethylethylenediamine (TMEDA) as a bidentate complexing ligand and under a continuous flow of $\mathrm{O}_{2}$ in dichloromethane, ${ }^{55}$ failed to yield detectable amounts of the coupled product (Table 2, entry 5). A strong dependency between the extent of conversion and solvent was found. When acetonitrile was used (method D; $\mathrm{CuCl}$, TMEDA, $\mathrm{O}_{2}$ ) the desired surface product SAM-2 was obtained with $0.3 \%$ of the surface acetylenes coupled (Table 2, entry 6 and Fig. 2a). It can thus be argued that stabilization of copper(I) oxidation state by strongly coordinating solvents, such as acetonitrile, is responsible for the success of these conditions. The protocol was checked using air as the oxidant providing similar extent of conversion (Table 2, entry 7). Moreover, it was observed that the addition of the strong base 1,4-diazabicyclo[2.2.2]octane (DABCO) was mandatory for successfully performing the reaction. As shown in Fig. 2b, coupling efficiencies rose to $0.9 \%$ (Table 2 , entry 8 ) following the protocol of method $\mathrm{E}$ $\left(\mathrm{CuCl}\right.$, TMEDA, DABCO, air). ${ }^{56}$ The replacement of 

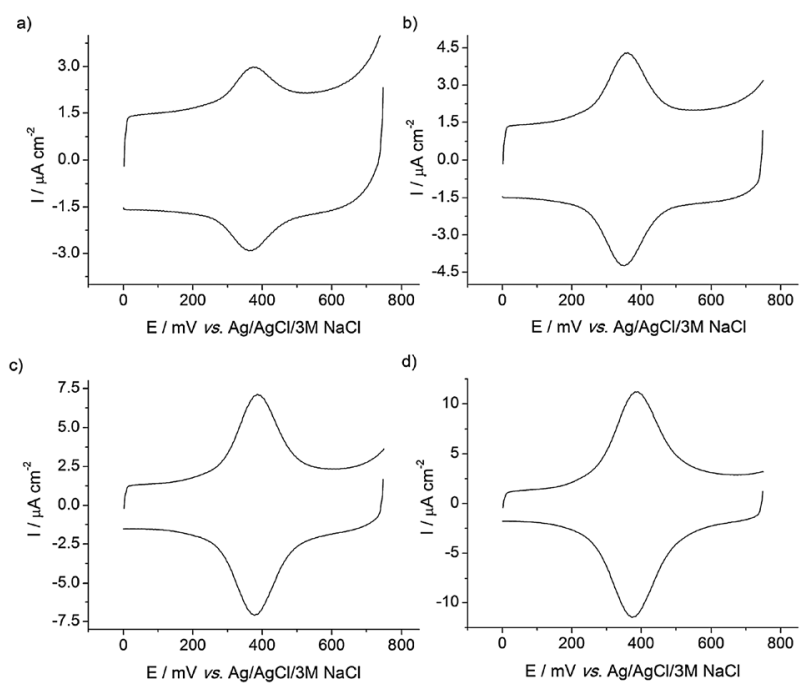

Fig. 2 Representative cyclic voltammograms of ethynylferrocenefunctionalized $\mathrm{Si}(100)$ surfaces (SAM-2). (a) Linkage prepared as for method D (Table 2, entry 6), (b) Surface prepared using oxidative coupling in the presence of DABCO (13 equiv) (Method E, Table 2, entry 8). (c-d) Optimized reaction conditions for oxidative acetylenic coupling at a $\mathrm{Si}(100)$ surface (Method F, Table 2, entries 10 and 13). Scan rate was $500 \mathrm{mV} \mathrm{s}^{-1}$ in $1.0 \mathrm{M} \mathrm{HClO}_{4}$ electrolyte.

DABCO by a stronger base, 1,8-diazabicyclo[5.4.0]undec7-ene (DBU), had no beneficial effect on the extent of conversion of SAM-1 (Table 2, entry 9). Deprotonation is seldom, if ever, a limiting step in oxidative couplings, because in the presence of copper the acidity of acetylenic $\mathrm{C}-\mathrm{H}$ is significantly increased. The role of the base is to merely act as a proton sink (a thermodynamic event), not to increase the rate of the deprotonation step (a kinetic consideration). Interestingly, provided that $\mathrm{DABCO}$ was present in the reaction mixture, the removal of the bidentate ligand TMEDA (method F, Fig. 2c) did result in marginally improved reaction yields (Table 2, entry 10), but replacement of $\mathrm{CuCl}$ for $\mathrm{CuI}$ had negligible effects on the outcome of coupling process (not shown). Efforts to further improve coupling efficiencies were made using this catalyst/base combination $(\mathrm{CuCl}, \mathrm{DABCO})$. Investigations were initially made for the choice of reaction times, concentrations of alkyne $\mathbf{2}$, catalyst and base loading. Finally, as a representative CV in Fig. 2d illustrates, optimum reaction conditions were achieved when a mixture of ethynylferrocene 2 ( $20 \mathrm{mM}), \mathrm{CuCl}$ ( 0.1 equiv), and $\mathrm{DABCO}$ ( 1.1 equiv) in acetonitrile was reacted in air for $24 \mathrm{~h}$ at a room temperature of $c a .23{ }^{\circ} \mathrm{C}$. This afforded the desired SAM-2 with an extent of reaction of the surface alkyne moieties of $c a .5 \%$ (Table 2, entry 13). Performing the heterocoupling reaction multiple times on the same silicon sample (Fig. S5, ESI $\dagger$ ) did result in only marginally improved yields ( $c a .9 \%$ ). This data suggests that the homocoupling of ferrocene 2 in solution competes poorly with the surface reaction and is not a major factor in preventing a higher extent of conversion. Although a low yield, for most surface electrochemistry studies low coverage of redox species are required to ensure the redox species do not interact and hence the low yield is not a drawback for our purposes. Several control experiments were conducted and results are summarized in Table 2 (entries 14-16).
Most importantly, when vinylferrocene (an olefin) and SAM-1 (acetylene) were reacted under the conditions of method $\mathrm{F}$ no reaction product was detected. ${ }^{57}$

\subsection{Spectroscopic and electrochemical characterization of redox 1,3-diyne assemblies on $\mathrm{Si}(100)$ electrodes}

The reflectivities from SAM-2 (Cu-catalyzed reaction) were simulated using a one-layer model. There was insignificant improvement in fit over a single layer to suggest a second layer (i.e. two-layer model). X-ray reflectivity data for surface SAM-2, formed by the reaction of SAM-1 with compound $\mathbf{2}$ (method F, Table 2, entry 13) revealed a thinner film (12.0(9) ^) than could be expected after the attachment of substituted ferrocene 2 . Although the SLD for this film $\left(14.9(1) \times 10^{-6} \AA^{-2}\right.$ ) is consistent with a fully dense monolayer, the relatively large roughness $(\sigma=7.8(1) \AA)$ in conjunction with the refined thickness indicate a low coupling efficiency $(<10 \%)$.

Due to the attractive electrochemical characteristics exhibited by ferrocene (i.e. fast electron-transfer rate, low oxidation potential, and stability) its covalent attachment by either ester, amide or 1,2,3-triazole linkages onto conducting surfaces has been widely reported..$^{21 c, 51,58}$ The redox 1,3-diyne derivatives prepared in this study exhibit stable cyclic voltammetric peaks attributable to the ferrocene/ferricenium reaction and surface coverages, $\Gamma$, ranging from $c a .0 .2$ to $c a .4 .0 \times 10^{-11} \mathrm{~mol} \mathrm{~cm}^{-2}$ (Table $\mathrm{S} 1$ in ESI $\dagger$ ). As introduced in the previous paragraph, on the basis of the electrochemical data, and from the perspective that surface acetylene groups have a footprint of 19(3) $\AA^{2}$ (refinement of XRR structural model for SAM-1), conversions of surface alkynes for this first example of oxidative acetylenic coupling at a surface were approximately $0.3-5 \%$.

One striking aspect of voltammograms obtained for $\mathrm{Si}(100)$ electrodes after the attachment of the acetylene bearing redox species 2 is the close-to-ideal redox response. ${ }^{59}$ Fig. 2 and Fig. 3 show representative cyclic voltammograms (CVs) of silicon electrodes incorporating the 1,3-diyne linker after coupling with ethynylferrocene 2 (SAM-2). An electrochemical summary is presented in Table $\mathrm{S} 1$ (ESI $\dagger)$. The values of peak widths at half maximum $\left(\Delta E_{\mathrm{fwhm}}\right)$ were as low as $104.7 \pm 0.3 \mathrm{mV}$ at a sweep rate of $100 \mathrm{mV} \mathrm{s}^{-1}$. $^{60}$ These values are only marginally larger than the theoretical (entropically determined) value of $91 \mathrm{mV}$, expected for identical and independent redox sites at room temperature. ${ }^{59,61} \mathrm{We}$ also note that the presence of appreciable repulsive lateral interactions among ferrocenyl units is not considered to be likely due to the small measured values of $\Gamma$. Hence it can be proposed that the observed redox centres in SAM-2 are effectively independent, i.e. nearly fully solvated by aqueous electrolytes and the local environment around each redox centre is remarkably uniform. ${ }^{58 c, 62}$

Peak currents in the voltammograms (Fig. S6, ESI $\dagger$ ) were found to scale linearly with the potential scan rate, $\nu$, rather than with $\nu^{1 / 2}$. These measurements were diagnostic of thinfilm behaviour and supported the formation of surface SAM-2. The half wave potential, $E_{1 / 2}$, extrapolated from voltammograms taken at low scan rates $\left(\nu=100 \mathrm{mV} \mathrm{s}^{-1}\right)$ was $373 \pm 13 \mathrm{mV}$ (Table S1), which is the same as the $c a .370 \mathrm{mV}$ value that we 


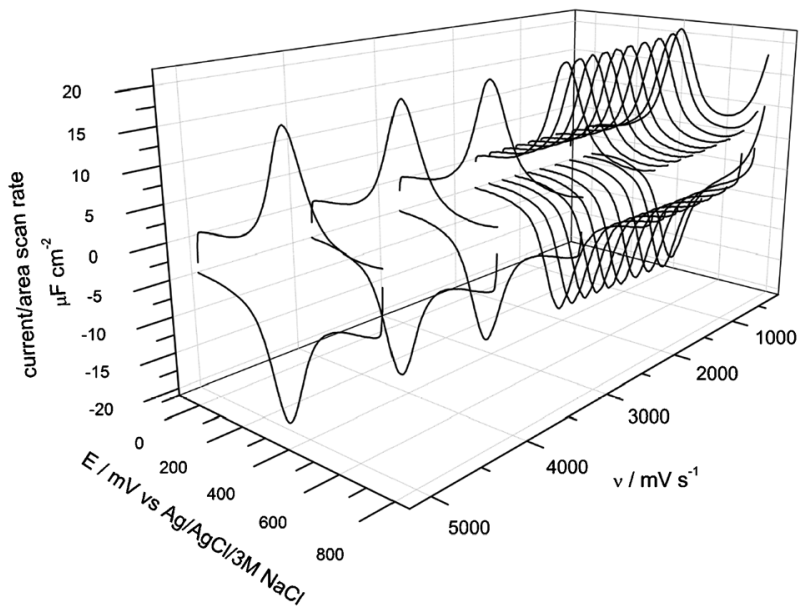

Fig. 3 Cyclic voltammograms of SAM-2 electrodes (method F) recorded at different scan rates $\left(\nu, 100-5000 \mathrm{mV} \mathrm{s}^{-1}\right)$. Currents were normalized to electrode area and scan rate.

and others have recently reported for other ferrocene-modified $\mathrm{Si}(100)$ and $\mathrm{Si}(111)$ electrodes. ${ }^{30 c, 63}$

We note that the estimated $E_{1 / 2}$ value for the 1,3-diyne derivative (SAM-2) is some $200 \mathrm{mV}$ more negative than the value for ethynylferrocene $\mathbf{2}$ directly grafted onto either $\mathrm{H}-\mathrm{Si}(100),{ }^{49 a, 64}$ or azide-terminated $\mathrm{Si}(100)$ monolayers (i.e. tethered [1,2,3]-triazoles). ${ }^{58 a}$

The nearly ideal voltammetry of alkyne 2 , attached to the acetylene monolayer via the non-polar 1,4-diyne linkage (ca. $100 \mathrm{mV}$ full width at half maximum), is also reminiscent of the nearly ideal voltammetry observed for the polar ester linkage in early examples of co-absorbed systems reported by Mujsce and co-workers. ${ }^{58 c}$ In that work, a direct, nonpolar methylene $\left(-\mathrm{CH}_{2}-\right)$ linkage to the ferrocene was also examined and shown to result in much broader, non-ideal voltammograms, even at a surface coverage as low as $10 \%$. Mujsce and co-workers hypothesized that the more polar linkage prevented the formation of aggregates of redox species, while favoring and ordered SAM packing. Quite surprisingly, it would thus appear that the 1,4-diyne linkage has a desirable mixture of conjugation and polarity that could prove useful in the context of assembling well-behaved electrode surfaces. We remark that as long as the appropriate reaction conditions were maintained (methods D-F), CV and electrochemical impedance spectroscopy (EIS) responses were reproducible (Table S1). The SAMs are very stable so that the electroactive surface coverage shows only a minor decrease $(4.5 \pm 3.1 \%$ decrease in $\Gamma)^{65}$ after prolonged cycling (100 CV cycles, Fig. S7, ESI $\dagger$ ). Both peak position (i.e. the value of $E$ at the maximum current values) and peak dispersion $\left(\Delta E_{\mathrm{fwhm}}\right)$ remained unaltered during the analysis (Fig. S7, ESI $\dagger$ ).

Shown in Fig. S8 (ESI $\dagger$ ) are the results of XPS analysis of SAM-2 samples (method F). The high quality of the SAM was confirmed by the absence of substantial silicon oxide signal in the 102-104 eV region of the XPS spectra. ${ }^{66}$ Further, combined quantitative $\mathrm{C} 1 \mathrm{~s}$ and Fe $2 \mathrm{p}$ XPS data suggested an approximate $10 \%$ conversion of surface acetylene moieties to the 1,3-diyne product (SAM-2). These figures are only marginally higher than those derived from $\mathrm{CV}$ measurements $(c a .5 \%)$. The high apparent $\mathrm{Fe} / \mathrm{C}$ ratio $(0.01 \pm 0.005)^{67}$ can be accounted for by the fact that the iron atoms are situated at the top of the monolayer and hence have the shortest electron escape path.

To investigate the electrode reaction kinetics of SAM-2, and thus to gain an insight of the performances of the 1,3-diyne linkage in the context of preparing chemically well-defined electrodes for fast electron transfer (ET), EIS experiments were conducted. $^{68}$

EIS investigates the system at steady-state, introducing only minor sinusoidal perturbations, thus kinetic parameters are considered more reliable than kinetic values derived from CV-based methods. ${ }^{68 c, 69}$ Fig. $4(\bigcirc)$ is a Nyquist representation of the impedance data for SAM-2 at $E_{\mathrm{dc}}=E_{1 / 2}$ ( $E_{\mathrm{dc}}$, dc offset).

Representative Bode plots are included in the ESI $\dagger$ paragraph (Fig. S9). Applying the formal potential, determined from ac voltammetry experiments at high frequencies $\left(>10^{4}\right.$ $\mathrm{Hz}$ ), to the electrode forces SAM-2 to be at equilibrium. ${ }^{68 d}$ Impedance data are interpreted by fitting the data to equivalent circuit models using the complex non-linear least-squares (CNLS) technique included in the frequency-response analysis software. The equivalent circuit used to fit the experimental data, as well as representative best-fit values for the discrete circuit elements, is shown in Fig. 4. The relationship between the circuit elements $\left(C_{\mathrm{dl}}\right.$, double-layer capacitance; $C_{\mathrm{ads}}$, adsorption pseudo-capacitance; $R_{\mathrm{ct}}$, charge-transfer resistance) of the simple model Fig. 4 and the parameters characterizing a redox system of strongly adsorbed electroactive species has been previously described, ${ }^{68 c, d}$ and the electron-transfer rate can be simply expressed as $k_{\text {et }}^{\circ}=\left(2 R_{\mathrm{ct}} C_{\mathrm{ads}}\right)^{-1}$. We remark that both $C_{\mathrm{d} l}$ and $C_{\mathrm{ads}}$ did show a frequency-dependent capacitive
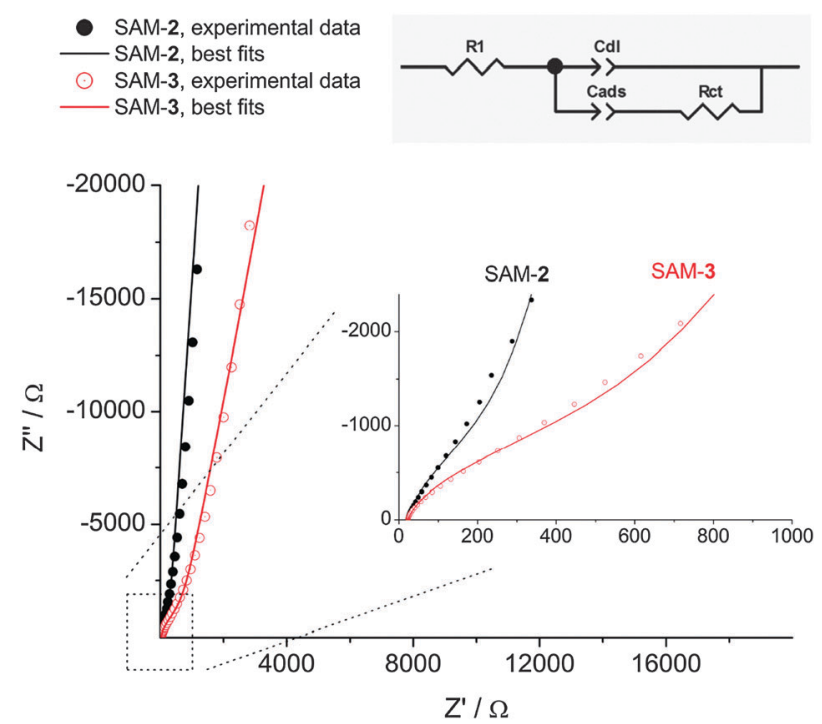

Fig. 4 Representative EIS Nyquist plots for SAM-2 (method F, entry 10 in Table 2) and SAM-3 at an applied potential $E_{\mathrm{dc}}=E_{1 / 2}$. Figure inset shows the high frequency range $(100 \mathrm{kHz}$ to $50 \mathrm{~Hz})$ of the spectra. EIS was interpreted by curve fitting the data to the equivalent circuit shown in figure. All symbols are experimental data, and solid lines are best fits to the data $\left(\chi^{2}<0.0015\right)$. The rate of charge transfer, $k^{\circ}$ et was $361.5 \mathrm{~s}^{-1}$ for SAM-2 $\left(R_{\mathrm{s}}=22.9 \Omega, C_{\mathrm{dl}}=1.10 \mu \mathrm{F}(\varphi=0.97)\right.$, $\left.C_{\text {ads }}=0.25 \mu \mathrm{F}(\varphi=0.99), R_{\mathrm{ct}}=5459 \Omega\right)$, and $123.2 \mathrm{~s}^{-1}$ for SAM-3 $\left(R_{\mathrm{s}}=22.8 \Omega, C_{\mathrm{dl}}=1.41 \mu \mathrm{F}(\varphi=0.94), C_{\mathrm{ads}}=1.13 \mu \mathrm{F}(\varphi=0.95)\right.$, $R_{\mathrm{ct}}=3601 \Omega$ ). 
behavior and were therefore treated as constant-phase elements (CPEs) for which the impedance is equal to $C(\mathrm{j} \omega)^{\phi}$, in which $C$ is the capacitance, $\omega$ is the angular frequency, and $\phi$ is an exponential term with a value between 0 and 1 . We note that through this report we treated CPEs as capacitors as the power-law modifier is typically between 0.94 and 0.99 , where 1.0 is an ideal capacitor. ${ }^{70}$ Using the refined $R_{\mathrm{ct}}$ and $C_{\mathrm{ads}}$ values, we obtained an apparent ET rate constant $\left(k_{\text {et }}^{\circ}\right)$ as high as $376 \pm 58 \mathrm{~s}^{-1}$ (method F; entry 10 in Table 2$)$. This value is moderately slower than the $500 \pm 2 \mathrm{~s}^{-1} k_{\text {et }}$ (electron-transfer relaxation rate constant ${ }^{71}$ ) reported by $\mathrm{Chidsey}^{58 b}$ for ethynylferrocene 2 SAMs on gold prepared using CuAAC reactions with mixed azide-terminated thiol/diluent thiol, but 4-fold faster than what observed for thinner films of ethynylferrocene 2 grafted directly onto $\mathrm{H}-\mathrm{Si}(100)$ substrates. $^{49 a}$

\subsection{A comparative ET study: 1,3-butadiyne vs. 1,2,3-triazole linkage}

Given the novelty of the use of oxidative acetylenic coupling in surface science, it is of interest to compare ET rates for SAM-2 samples with ET rates measured for redox SAMs prepared via well-established grafting protocols. Since being initially reported, ${ }^{2 c, d} \mathrm{CuAAC}$ reactions have been extensively explored by the surface science community. ${ }^{16 b, 72}$ The powerful combination of advantages offered by $\mathrm{CuAAC}$ reactions have allowed for numerous elegant redox chemistry applications at an electrode surface of the putative 1,4-disubstituted [1,2,3]triazole product. ${ }^{58 a, d, 73}$ Furthermore, triazoles provide electronic coupling comparable to that reported with other linkers. ${ }^{58 b}$ In this report azidomethylferrocene $\mathbf{3}$ was immobilized onto the acetylene layer to yield the "click" product (SAM-3) using a modification of a previously reported procedure (Scheme S1, ESI $\dagger){ }^{30 e}$ Due to the low extent of conversion of surface alkynes observed here in the formation of SAM-2 (Table 2 and Table S1) and considering the well-documented dependence of the rate constant, $k^{\circ}$ et, on surface coverage $(\Gamma),{ }^{58 c, 62 a}$ we considered for our comparative study only SAM-3 samples with $\Gamma$ values smaller than ca. $3-5 \times 10^{-11} \mathrm{~mol} \mathrm{~cm}^{-2} \cdot 74$ Representative cyclic voltammograms for surface SAM-3 samples are shown in Fig. S10 (ESI $\dagger)$. The voltammetric response of these electrodes was close to ideal and displayed a remarkable stability with a decrease in $\Gamma$ of $5.2 \pm 3.4 \%$ after $100 \mathrm{CV}$ cycles (Fig. S11, ESI $\dagger$ ). ${ }^{75}$

ET rates show an exponential distance dependence with decay constants typically about $1.0 \AA^{-1}$ for electron transfer trough saturated chains, ${ }^{76}$ and 0.2 to $0.6 \AA^{-1}$ for unsaturated spacers. ${ }^{77}$ Despite their chemically distinct composition, a fortunate match in the calculated distance (Fig. S12, ESI $\dagger$ ) separating the silicon substrate to cyclopentadienyl ring in both SAM-2 and SAM-3, allowed for a more straightforward comparison of $k_{\text {et }}^{\circ}$ values. ${ }^{78}$ Further, kinetics of charge transfer at an electrode surface are known to be influenced by a range of factors (e.g. electrolyte type and concentrations, ${ }^{79}$ length/ chemical nature of diluents molecules ${ }^{80}$ ) that were left unaltered in SAM-2 and SAM-3. As shown in Fig. $4(O)$ and in Fig. S9, and as summarized in Table S1, electron transfer was significantly faster (factor of 2.2-3.2) in monolayers with a 1,3-disubstituted diyne linkage (SAM-2) relative to that in monolayers with a 1,3-disubstituted [1,2,3]-triazole (SAM-3). The effect is not that unexpected as the electrontransfer rate depends strongly on the length and degree of conjugation of the organic spacer separating the electrode to tethered redox unit. ${ }^{58 b}$ Moreover, a marked difference in reorganization energy between ferrocenyl units in SAM-2 and SAM-3 is not a likely explanation for this dramatic difference in rates.

\section{Conclusions}

We have demonstrated that $\mathrm{Cu}^{\mathrm{I}}$-catalyzed oxidative acetylene coupling reactions can be employed to construct organic structures with fast electron transfer properties on $\mathrm{Si}(100)$ surfaces. The reaction proceeds in the time scale of hours using inexpensive catalysts and under non-stringent oxidative conditions. No activation reactions are required. In this manner, acetylene-terminated alkenyl monolayers on nonoxidized silicon can be functionalized with substituted alkynes in a short synthetic sequence providing a novel tool for integrating molecular structures with Si-based electronics. Our preliminary results on the acetylenic coupling involving tethered terminal alkynes remain empirical although the reaction has been carefully optimized in solution, and is well understood at a mechanistic level in this context. ${ }^{2 h}$ Further studies are needed to improve reaction yields, but nevertheless we believe there will be novel and more sophisticated applications of acetylenic coupling reactions at electrode surfaces, as this report unambiguously shows the ease with which functionality can be introduced with the alkyne/alkyne coupling and the superior electronic coupling properties of the 1,3-diyne linkage.

\section{Acknowledgements}

This research was supported by the Australian research Council's Discovery Projects Funding Scheme (project number DP01094564 and DP0556397).

\section{Notes and references}

1 (a) Modern Acetylene Chemistry, ed. P. J. Stang and F. Diederich, WILEY-VCH, Weinheim, 1995; (b) L. Brandsma, Preparative Acetylenic Chemistry, Elsevier Science Publishers B. V., New York, 1988

2 (a) K. H. Dötz, Angew. Chem., Int. Ed. Engl., 1975, 14, 644-645; (b) R. Huisgen, Pure Appl. Chem., 1989, 61, 613-628; (c) V. V. Rostovtsev, L. G. Green, V. V. Fokin and K. B. Sharpless, Angew. Chem., Int. Ed., 2002, 41, 2596-2599; (d) C. W. Tornøe, C. Christensen and M. Meldal, J. Org. Chem., 2002, 67, 3057-3064; (e) R. Chinchilla and C. Nájera, Chem. Rev., 2007, 107, 874-922; (f) K. Sonogashira, Y. Tohda and N. Hagihara, Tetrahedron Lett., 1975, 16, 4467-4470; (g) T. Baba, H. Kizuka, H. Handa and Y. Ono, Appl. Catal., A 2000, 194-195, 203-211; (h) P. Siemsen, R. C. Livingston and F. Diederich, Angew. Chem., Int. Ed., 2000, 39, 2632-2657; (i) B. Z. Tang, Macromol. Chem. Phys., 2008, 209, 1303-1307; (j) H. A. Dieck and F. R. Heck, J. Organomet. Chem., 1975, 93, 259-263; (k) E. Negishi, Acc. Chem. Res., 1982, 15, 340-348; (l) J. A. Marsden, J. J. Miller and M. M. Haley, Angew. Chem., Int. Ed., 2004, 43, 1694-1697.

3 F.-T. Luo and W. Ren-Tzong, Tetrahedron Lett., 1993, 34, 5911-5914.

4 H. Hofmeister, K. Annen, H. Laurent and R. Wiechert, Angew. Chem., Int. Ed. Engl., 1984, 23, 727-729. 
5 P. J. Stang, Angew. Chem., Int. Ed. Engl., 1992, 31, 274-285.

6 M. Regitz, Chem. Rev., 1990, 90, 191-213.

7 (a) Y. Wakatsuki, Z. Hou and M. Tokunaga, Chem. Rec., 2003, 3, 144-157; (b) F. Alonso, I. P. Beletskaya and M. Yus, Chem. Rev., 2004, 104, 3079-3160.

8 L. Shu, Z. Mu, H. Fuchs, L. Chi and M. Mayor, Chem. Commun., 2006, 1862-1863.

9 C. Li and Y. Li, Macromol. Chem. Phys., 2008, 209, 1541-1552.

10 A. Nantalaksakul, D. Reddy, C. Bardeen and S. Thayumanavan, Photosynth. Res., 2006, 87, 133-150.

11 A. Vessières, S. Top, C. Vaillant, D. Osella, J.-P. Mornon and G. Jaouen, Angew. Chem., Int. Ed. Engl., 1992, 31, 753-755.

12 J. Y. Wong, R. Langer and D. E. Ingber, Proc. Natl. Acad. Sci. U. S. A., 1994, 91, 3201-3204.

13 A. L. Smith and K. C. Nicolaou, J. Med. Chem., 1996, 39, 2103-2117.

14 D. Venkataraman, S. Lee, J. Zhang and J. S. Moore, Nature, 1994, 371, 591-593.

15 Modern Acetylene Chemistry, ed. P. J. Stang and F. Diederich, VCH, Weinheim, 1995.

16 (a) V. D. Bock, H. Hiemstra and J. H. van Maarseveen, Eur. J. Org. Chem., 2006, 51-68; (b) N. K. Devaraj and J. P. Collman, QSAR Comb. Sci., 2007, 26, 1253-1260; (c) Y. Li, J. Wang and C. Cai, Langmuir, 2011, 27, 2437-2445.

17 (a) J. K. Young, J. C. Nelson and J. S. Moore, J. Am. Chem. Soc., 1994, 116, 10841-10842; (b) M. Erdelyi and A. Gogoll, J. Org. Chem., 2003, 68, 6431-6434; (c) M. Rist, N. Amann and H.-A. Wagenknecht, Eur. J. Org. Chem., 2003, 2498-2504; (d) J. Li, P. S. Thiara and M. Mrksich, Langmuir, 2007, 23, 11826-11835; (e) J.-C. Lin, J.-H. Kim, J. A. Kellar, M. C. Hersam, S. T. Nguyen and M. J. Bedzyk, Langmuir, 2010, 26, 3771-3773.

18 (a) D. Wayner and R. Wolkow, J. Chem. Soc., Perkin Trans. 2, 2002, 23-34; (b) J. M. Buriak, Chem. Commun., 1999, 1051-1060; (c) J. M. Buriak, Chem. Rev., 2002, 102, 1271-1308; (d) R. Boukherroub, Curr. Opin. Solid State Mater. Sci., 2005, 9, 66-72; (e) N. Shirahata, A. Hozumi and T. Yonezawa, Chem. Rec., 2005, 5, 145-159.

19 (a) M. R. Linford, P. Fenter, P. M. Eisenberger and C. E. D. Chidsey, J. Am. Chem. Soc., 1995, 117, 3145-3155; (b) S. R. Puniredd, O. Assad and H. Haick, J. Am. Chem. Soc., 2008, 130, 13727-13734; (c) A. B. Sieval, A. L. Demirel, J. W. M. Nissink, M. R. Linford, J. H. van der Maas, W. H. de Jeu, H. Zuilhof and E. J. R. Sudhölter, Langmuir, 1998, 14, 1759-1768; (d) M. M. Sung, G. J. Kluth, O. W. Yauw and R. Maboudian, Langmuir, 1997, 13, 6164-6168.

20 R. J. Hamers, Annu. Rev. Anal. Chem., 2008, 1, 707-736.

21 (a) L. Scheres, A. Arafat and H. Zuilhof, Langmuir, 2007, 23, 8343-8346; (b) L. Scheres, M. Giesbers and H. Zuilhof, Langmuir, 2010, 26, 10924-10929; (c) D. Zigah, C. Herrier, L. Scheres, M. Giesbers, B. Fabre, P. Hapiot and H. Zuilhof, Angew. Chem., Int. Ed., 2010, 49, 3157-3160.

22 C. Coletti, A. Marrone, G. Giorgi, A. Sgamellotti, G. Cerofolini and N. Re, Langmuir, 2006, 22, 9949-9956.

23 P. Ball, Nat. Mater., 2005, 4, 119-119.

24 M. Y. Simmons, F. J. Ruess, K. E. J. Goh, W. Pok, T. Hallam, M. J. Butcher, T. C. G. Reusch, G. Scappucci, A. R. Hamilton and L. Oberbeck, Int. J. Nanotechnol., 2008, 5, 352-369.

25 (a) B. Fabre, Acc. Chem. Res., 2010, 43, 1509-1518; (b) A. Vilan, O. Yaffe, A. Biller, A. Salomon, A. Kahn and D. Cahen, $A d v$. Mater., 2010, 22, 140-159.

26 (a) T. Strother, R. J. Hamers and L. M. Smith, Nucleic Acids Res., 2000, 28, 3535-3541; (b) F. Wei, B. Sun, Y. Guo and X. S. Zhao, Biosens. Bioelectron., 2003, 18, 1157-1163; (c) R. Voicu, R. Boukherroub, V. Bartzoka, T. Ward, J. T. C. Wojtyk and D. D. M. Wayner, Langmuir, 2004, 20, 11713-11720; (d) H. B. Yin, T. Brown, R. Greef, J. S. Wilkinson and T. Melvin, Microelectron. Eng., 2004, 73-74, 830-836; (e) W. Cai, J. R. Peck, D. W. van der Weide and R. J. Hamers, Biosens. Bioelectron., 2004, 19, 1013-1019; (f) G. Qin, C. Santos, W. Zhang, Y. Li, A. Kumar, U. J. Erasquin, K. Liu, P. Muradov, B. W. Trautner and C. Cai, J. Am. Chem. Soc., 2010, 132, 16432-16441.

27 (a) J. M. Tour, Chem. Rev., 1996, 96, 537-554; (b) J. A. Marsden and M. M. Haley, J. Org. Chem., 2005, 70, 10213-10226.

28 A. S. Hay, J. Org. Chem., 1962, 27, 3320-3321.
29 G. Eglinton and A. R. Galbraith, J. Chem. Soc., 1959, 889-896.

30 (a) S. Ciampi, T. Böcking, K. A. Kilian, J. B. Harper and J. J. Gooding, Langmuir, 2008, 24, 5888-5892; (b) S. Ciampi, T. Böcking, K. A. Kilian, M. James, J. B. Harper and J. J. Gooding, Langmuir, 2007, 23, 9320-9329; (c) S. Ciampi, P. K. Eggers, G. Le Saux, M. James, J. B. Harper and J. J. Gooding, Langmuir, 2009, 25, 2530-2539; (d) S. Ciampi and J. J. Gooding, Chem.-Eur. J., 2010, 16, 5961-5968; (e) S. Ciampi, G. Le Saux, J. B. Harper and J. J. Gooding, Electroanalysis, 2008, 20, 1513-1519; $(f)$ A. Ng, S. Ciampi, M. James, J. B. Harper and J. J. Gooding, Langmuir, 2009, 25, 13934-13941; (g) H. Liu, F. Duclairoir, B. Fleury, L. Dubois, Y. Chenavier and J.-C. Marchon, Dalton Trans., 2009, 3793-3799; (h) M. James, T. A. Darwish, S. Ciampi, S. O. Sylvester, Z. Zhang, A. Ng, J. J. Gooding and T. L. Hanley, Soft Matter, 2011, 7, 5309-5318.

31 (a) B. Guan, S. Ciampi, G. Le Saux, K. Gaus, P. J. Reece and J. J. Gooding, Langmuir, 2011, 27, 328-334; (b) B. Guan, A. Magenau, K. A. Kilian, S. Ciampi, K. Gaus, P. J. Reece and J. J. Gooding, Faraday Discuss., 2011, 149, 301-317; (c) S. Ciampi, M. James, P. Michaels and J. J. Gooding, Langmuir, 2011, 27, 6940-6949.

32 (a) P. F. H. Schwab, M. D. Levin and J. Michl, Chem. Rev., 1999, 99, 1863-1934; (b) P. T. Hurley, A. E. Ribbe and J. M. Buriak, J. Am. Chem. Soc., 2003, 125, 11334-11339; (c) G. Z. Liu and J. J. Gooding, Langmuir, 2006, 22, 7421-7430.

33 We note that covalent attachment of unactivated 1-alkynes onto $\mathrm{Si}(111)-\mathrm{H}$ has been recently reported to occur at room temperature and in the dark (ref. 21a-b). Nevertheless, narrow Si $2 \mathrm{p}$ scans showed a decreasing $\mathrm{Si} 2 \mathrm{p}$ signal with increased reaction temperature (ref. 21a), which is attributed to the increased coverage of the silicon substrate by the 1-alkyne molecule.

34 J. E. Baio, T. Weidner, J. Brison, D. J. Graham, L. J. Gamble and D. G. Castner, J. Electron Spectrosc. Relat. Phenom., 2009, 172, 2-8.

35 (a) L. J. Webb and N. S. Lewis, J. Phys. Chem. B, 2003, 107, 5404-5412; (b) A. B. Sieval, R. Linke, H. Zuilhof and E. J. R. Sudhölter, Adv. Mater., 2000, 12, 1457-1460; (c) P. T. Hurley, E. J. Nemanick, B. S. Brunschwig and N. S. Lewis, J. Am. Chem. Soc., 2006, 128, 9990-9991.

36 Under our XPS experimental conditions the $\mathrm{SiO}_{x}$ sublayer detection limit was approximated to 0.06 monolayers.

37 (a) O. Seitz, T. Böcking, A. Salomon, J. J. Gooding and D. Cahen, Langmuir, 2006, 22, 6915-6922; (b) F. J. Himpsel, F. R. McFeely, A. Taleb-Ibrahimi, J. A. Yarmoff and G. Hollinger, Phys. Rev. B, 1988, 38, 6084-6096.

38 SLD for X-rays is obtained by multiplying the electron density $\left(\mathrm{e}^{-} / \AA^{3}\right)$ of the material by the factor $2.82 \times 10^{-5} \AA$.

39 A. Nelson, J. Appl. Crystallogr., 2006, 39, 273-276.

40 Estimated standard deviations (esd's) are given in parentheses.

41 T. Böcking, M. James, H. G. L. Coster, T. C. Chilcott and K. D. Barrow, Langmuir, 2004, 20, 9227-9235.

42 H. Jin, C. R. Kinser, P. A. Bertin, D. E. Kramer, J. A. Libera, M. C. Hersam, S. T. Nguyen and M. J. Bedzyk, Langmuir, 2004, 20, $6252-6258$.

43 E. L. S. Wong, M. James, T. C. Chilcott and H. G. L. Coster, Surf. Sci., 2007, 601, 5740-5743.

44 (a) W. Chodkiewicz, Ann. Chim. (Paris), 1957, 2, 819-869; (b) J. P. Marino and H. N. Nguyen, J. Org. Chem., 2002, 67, 6841-6844.

45 J.-H. Li, Y. Liang and Y.-X. Xie, J. Org. Chem., 2005, 70, 4393-4396.

46 Ref. $2 h$ has reviewed in detail the scope and limitations of both the copper- and palladium-catalyzed homocoupling reactions.

47 The iron(III) emission was $c a .6 \%$ of the total iron Fe $2 p$ signal.

48 R. Zanoni, A. Aurora, F. Cattaruzza, C. Coluzza, E. A. Dalchiele, F. Decker, G. Di Santo, A. Flamini, L. Funari and A. G. Marrani, Mater. Sci. Eng., 2006, 26, 840-845.

49 (a) A. G. Marrani, F. Cattaruzza, F. Decker, R. Zanoni, M. Cossi and M. F. Iozzi, J. Nanosci. Nanotechnol., 2010, 10, 2901-2907; (b) R. Zanoni, F. Cattaruzza, C. Coluzza, E. A. Dalchiele, F. Decker, G. Di Santo, A. Flamini, L. Funari and A. G. Marrani, Surf. Sci., 2005, 575, 260-272.

50 X. G. Zhang, Electrochemistry of Silicon and its Oxide, Kluwer/ Plenum, New York, 2001.

51 B. Fabre and F. Hauquier, J. Phys. Chem. B, 2006, 110, 6848-6855. 
52 (a) K. Kowalski and S. Domagala, J. Organomet. Chem., 2007, 692, 3100-3103; (b) J.-G. Rodriguez, A. Oñate, R. M. MartinVillamil and I. Fonseca, J. Organomet. Chem., 1996, 513, 71-76.

53 U. H. F. Bunz, G. Roidl, M. Altmann, V. Enkelmann and K. D. Shimizu, J. Am. Chem. Soc., 1999, 121, 10719-10726.

54 Extent of conversion for the derivatization of surface acetylenes (SAM-1) as low as $0.1 \%$ could be determined accurately with via electrochemical measurements well above detection limits.

55 The latter protocol $\left(\mathrm{O}_{2}, \mathrm{CuCl}, \mathrm{TMEDA}\right)$ is commonly referred to as standard Hay coupling conditions.

56 L. Fomina, B. Vazquez, E. Tkatchouk and S. Fomine, Tetrahedron, 2002, 58, 6741-6747.

57 The coupling of unactivated olefins and acetylenes mediated by transition metal complexes (e.g. Ru complexes) has been the subject of recent investigation (C. Slugovc, K. Mereiter, R. Schmid and K. Kirchner, Organometallics, 1999, 18, 1011-1017). Nevertheless, the reaction conditions of method F seems to favor acetylenic heterocoupling reactions versus olefinicacetylenic coupling.

58 (a) A. G. Marrani, E. A. Dalchiele, R. Zanoni, F. Decker, F. Cattaruzza, D. Bonifazi and M. Prato, Electrochim. Acta, 2008， 53, 3903-3909; (b) N. K. Devaraj, R. A. Decreau, W. Ebina, J. P. Collman and C. E. D. Chidsey, J. Phys. Chem. B, 2006, 110, 15955-15962; (c) C. E. D. Chidsey, C. R. Bertozzi, T. M. Putvinski and A. M. Mujsce, J. Am. Chem. Soc., 1990, 112, 4301-4306; (d) J. P. Collman, N. K. Devaraj, T. P. A. Eberspacher and C. E. D. Chidsey, Langmuir, 2006, 22, 2457-2464; (e) G. Liu, J. Liu, T. Böcking, P. K. Eggers and J. J. Gooding, Chem. Phys., 2005, 319, 136-146.

59 E. Laviron, J. Electroanal. Chem., 1979, 101, 19-28.

60 Only a minor increase $(<10 \%)$ of the half width values was generally observed at scan rates as high as $5000 \mathrm{mV} \mathrm{s}^{-1}$.

61 P. Daum and R. W. Murray, J. Electroanal. Chem., 1979, 103, 289-294.

62 (a) J. J. Calvente, R. Andreu, M. Molero, G. Lopez-Perez and M. Dominguez, J. Phys. Chem. B, 2001, 105, 9557-9568; (b) G. K. Rowe and S. E. Creager, Langmuir, 1991, 7, 2307-2312.

63 N. Tajimi, H. Sano, K. Murase, K.-H. Lee and H. Sugimura, Langmuir, 2007, 23, 3193-3198.

64 M. Cossi, M. F. Iozzi, A. G. Marrani, T. Lavecchia, P. Galloni, R. Zanoni and F. Decker, J. Phys. Chem. B, 2006, 110, 22961-22965.

65 Data obtained from the analysis of 15 independently prepared and analyzed electrodes (SAM-2).
66 The approximate fractional monolayer coverage of $\mathrm{SiO}_{x}$ varied from not detectable $(<0.06)$ to 0.1 monolayers equivalents.

67 Data obtained from the XPS analysis of 6 independently prepared and analyzed surface samples (SAM-2).

68 (a) S. Song, R. A. Clark, E. F. Bowden and M. J. Tarlov, J. Phys. Chem., 1993, 97, 6564-6572; (b) J. Liu, Y. Jin, A. Wu, Z. Li and S. Dong, Electroanalysis, 2004, 16, 1931-1937; (c) A. D. Abhayawardhana and T. C. Sutherland, J. Phys. Chem. C, 2009, 113, 4915-4924; (d) S. E. Creager and T. T. Wooster, Anal. Chem., $1998,70,4257-4263$

69 S. A. Trammell, D. S. Seferos, M. Moore, D. A. Lowy, G. C. Bazan, J. G. Kushmerick and N. Lebedev, Langmuir, 2007, 23, 942-948.

70 P. Zoltowski, J. Electroanal. Chem., 1998, 443, 149-154.

71 Electron-transfer relaxation rate constants were here determined from the current transient following a potential step $\left(\mathrm{E}^{\circ}-10 \mathrm{mV}\right.$ to $\left.\mathrm{E}^{\circ}\right)$.

72 S. Ciampi, J. B. Harper and J. J. Gooding, Chem. Soc. Rev., 2010, 39, 2158-2183.

73 (a) J. P. Collman, N. K. Devaraj and C. E. D. Chidsey, Langmuir, 2004, 20, 1051-1053; (b) N. K. Devaraj, P. H. Dinolfo, C. E. D. Chidsey and J. P. Collman, J. Am. Chem. Soc., 2006, 128, 1794-1795; (c) R. A. Decréau, J. P. Collman and A. Hosseini, Chem. Soc. Rev., 2010, 39, 1291-1301.

74 The "click" immobilization of azide $\mathbf{3}$ is a highly efficient coupling procedure, therefore, in order to maintain low $\Gamma$ values, very short reaction times and low concentrations of azide were necessary.

75 Data obtained from the analysis of 5 independently prepared and analyzed electrodes (SAM-3).

76 J. F. Smalley, S. W. Feldberg, C. E. D. Chidsey, M. R. Linford, M. D. Newton and Y.-P. Liu, J. Phys. Chem., 1995, 99, 13141-13149.

77 S. B. Sachs, S. P. Dudek, R. P. Hsung, L. R. Sita, J. F. Smalley, M. D. Newton, S. W. Feldberg and C. E. D. Chidsey, J. Am. Chem. Soc., 1997, 119, 10563-10564.

78 From the analysis of energy-minimized structures (semi-empirical MOPAC calculation, Chem3D Ultra) the distance separating $\mathrm{Si}$ atop atoms and the $\mathrm{C}-1$ of the cyclopentadienyl ring were 1.45 and $1.40 \mathrm{~nm}$ for SAM-2 and SAM-3, respectively.

79 H. Ju and D. Leech, Phys. Chem. Chem. Phys., 1999, 1, 1549-1554.

80 (a) J. J. Sumner and S. E. Creager, J. Phys. Chem. B, 2001, 105, 8739-8745; (b) A. M. Napper, H. Liu and D. H. Waldeck, J. Phys Chem. B, 2001, 105, 7699-7707; (c) P. K. Eggers, D. B. Hibbert, M. N. Paddon-Row and J. J. Gooding, J. Phys. Chem. C, 2009, 113, 8964-8971. 